\title{
MICRORNA, A CLINICAL DIAGNOSTIC AND PROGNOSTIC BIOMARKER
}

\author{
Anitha TS, Scientist \\ Central Inter-Disciplinary Research Facility
}

Sri Balaii Vidyapeeth Mahatma Gandhi Medical College and Research Institute Campus

Pillaiyarkuppam, Puducherry - 607403, India

Email:tsanitha6@gmail.com

?

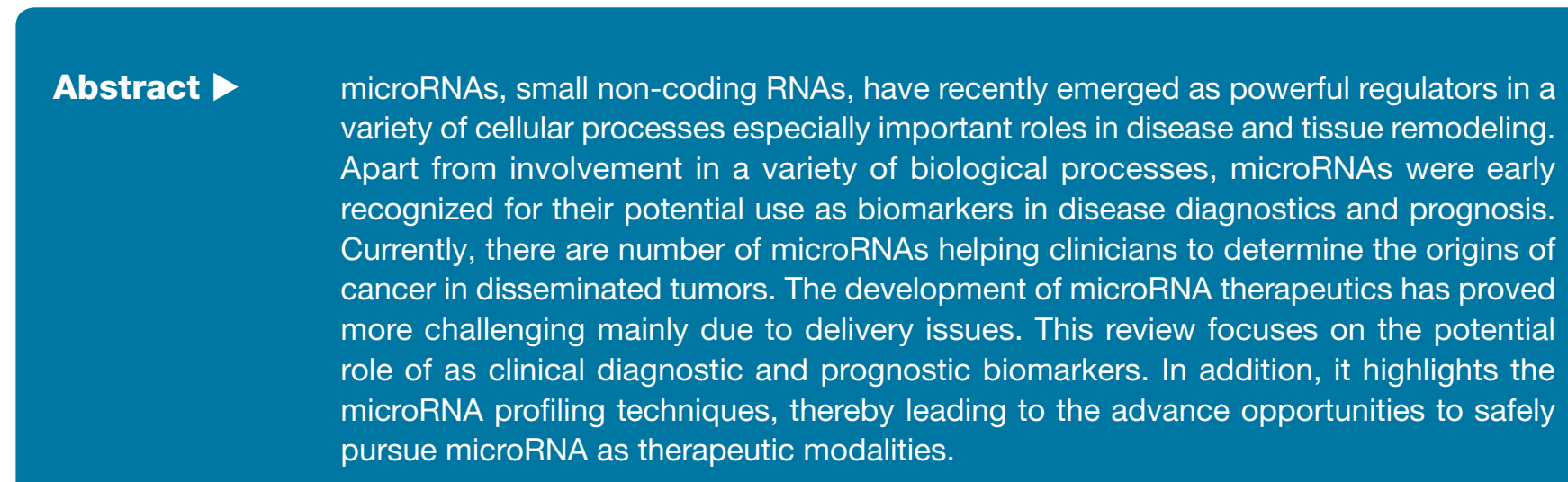

Key Words: miRNAs, biomarkers, prognostic markers, profiling, diabetes mellitus.

\section{Introduction}

microRNAs (miRNAs) are evolutionarily conserved small non-coding RNA molecules that regulate gene expression, and their recent discovery is revolutionizing both basic biomedical research and drug discovery. The human genome is believed to encode $\sim 1,000$ miRNAs. A repository of miRNAs from many organisms has been listed in the miRBase Sequence Database $^{1}$, that contains sequences and annotation ${ }^{2}$. More than 25,000 miRNAs have been described in man, worms, Drosophila, and also in the small plant Arabidopsis thaliana ${ }^{3}$. This review aims to describe the basics of miRNA and their role as biomarkers in clinical diagnostics and prognostic values. Moreover, this review also discusses the promising methods for measuring miRNA expression profiles in various biological samples such as cells, tissues and body fluids.

\section{miRNA basics}

Gary Ruvkun and Victor Ambros in 1990, first discovered miRNAs in Caenorbabditis elegans and their target gene $e^{4,5}$. Together, these two seminal discoveries identified a novel mechanism for post-transcriptional gene regulation. A hairpin fold-back structure from the precursor transcript separates the miRNAs from other small RNAs with expression confirmation of about 22 nucleotide-long mature sequence. Presently, based on deep-sequencing data, 1600 human miRNA precursors have been deposited into miRBase $v 19^{6}$. The nomenclature of these miRNAs is based on a "mir" or "miR" prefix with identifying numbers assigned sequentially at the time of discovery. "mir" represents a precursor miRNA whereas "miR" denotes a mature miRNA sequence. Similar or identical sequences can be given the same number. 
miRNA biogenesis

miRNA genes reside either in intergenic regions, within introns of coding or non-coding genes or within exons of non-coding genes ${ }^{7}$. Approximately one third of miRNAs are intergenic and most of all miRNA loci contain clustered miRNAs (miRBase v19). The majority of miRNAs are transcribed as long primary transcripts by RNA polymerase II and many are capped and polyadenylated ${ }^{8,9}$. Analysis of miRNAs residing in intergenic primary transcripts indicates that such transcripts are shorter than protein-coding transcripts with transcriptional start sites about $2 \mathrm{~kb}$ upstream of the pre-miRNA and polyadenylation signals $2 \mathrm{~kb}$ downstream ${ }^{10}$. A subset of miRNAs is transcribed by RNA polymerase III. This cluster of miRNAs is located among Alu rich regions on chromosome $19^{11}$. Pri-miRNAs fold into hairpin structures containing imperfectly base-paired stems and are processed into 60- to 100-nt hairpins known as pre-miRNAs. The pre-miRNAs are exported from the nucleus to the cytoplasm by exportin 5, where they, in general, are cleaved by the endonuclease Dicer to yield imperfect miRNA-miRNA* duplexes ${ }^{12}$. The miRNA strand is selected to become a mature miRNA, whereas, most often, the miRNA* strand is degraded. The mature miRNA that is added to the RNA-induced silencing complex (RISC) identifies the potential precise targets and activates post- transcriptional gene silencing ${ }^{13}$. On the other hand, an alternative biogenesis pathway was revealed in which miR-451 enters RISC by direct loading of the pre-miR into RISC after Drosha processing, by skipping further processing by Dicer ${ }^{14}$. Transcription of miRNAs occurs through RNA polymerase II and subsequent processing is mediated by the nuclear ribonuclease III (RNase III) enzyme Drosha to form precursor miRNAs (70-100 nucleotides). Following transportation to the cytoplasm by exportin 5 , a further cleavage occurs via another RNase III enzyme, Dicer, to form the mature miRNA. miRNAs modulate both physiological and pathological pathways by post-transcriptionally inhibiting the expression of a multitude of target genes. Much work has been done on the role of miRNAs in human disease, especially in cancers and infections.

Use of miRNA as biomarkers in clinical diagnosis

miRNAs exhibit strict developmental and tissuespecific expression patterns in organ and immune system development. For example, miR-1 is involved in mammalian heart development ${ }^{15}$, miR-375 regulates pancreatic insulin secretion ${ }^{16}$, miR-181 influences the differentiation of hematopoietic cells toward the $\mathrm{B}$-cell lineage $\mathrm{e}^{17}$, and $\mathrm{miR}-430$ is required for zebrafish brain development ${ }^{18}$. These studies highlight the participation of miRNAs in diverse cellular processes. Hence, it is not surprising that dysregulation of miRNA function is associated with many human diseases such as diabetes, neurological disorders, and cancer.

\section{miRNA as biomarker for cancer}

miRNAs play a critical role in the development of cancer and can influence cancer-promoting and cancersuppressing genes. The first documentation of a miRNA abnormality in cancer stemmed from studies of human chromosome $13 \mathrm{q} 14^{19}$. In cancer, miRNA expression variations are evident across different stages of cancer progression $^{20}$. In tumorigenesis, overexpression of certain miRNA down-regulates tumor suppressor genes. These miRNAs can be exploited as potential biomarkers due to their tissue specificity and to tumor type and its origin ${ }^{21}$. An increasing number of miRNAs are now identified and utilized as prognostic miRNAs to predict drug response.

\section{Glioblastoma}

The primary brain tumor, glioma arises from glial cells of the central nervous system (CNS). Even after aggressive treatment like surgical resection and chemotherapy, glioblastoma multiforme patients show the least promising prognosis, where the reappearance is frequent and mean survival is only $12-15$ months $^{22}$. The intricacy in determining an explicit biomarker for glioma lies in part with the complex heterogeneous nature of the cancer itself. miRNA signatures have been recognized in both glioblastoma tissue and in blood circulation of glioblastoma patients. Recently, deep sequencing method has produced one of the largest sets of miRNA profiles for glioblastoma and control brain tissue. This study identified 33 up-regulated miRNA in the glioblastoma tissue and 40 down-regulated. In addition, 18 novel miRNAs and 16 novel miRNA-3ps were identified (miRNA-3p, miR-3676, miR-204, miR-539, miR-758, miR-382, miR-1271miR-98, miR-1307, miR-181b1-miR-873, miR-212, miR-135a-2, miR511-1, miR-301a, miR-381, miR-487a) ${ }^{23}$.

\section{Breast cancer}

Profiling studies of miRNA have led to the categorization of miRNAs that are abnormally expressed in human breast 
cancer, with down regulation of $\mathrm{miR}-125 \mathrm{~b}, \mathrm{miR}-10 \mathrm{~b}$ and miR-145 and up-regulation of miR-21, miR-9 and miR-155. When comparing breast cancer tissue with the normal tissues, 29 differentially-expressed miRNAs were acknowledged, and a subset of $15 \mathrm{miRNAs}$ could be used to discriminate tumor from normal. In recent years, the discovery of upregulation of miR-10b in promoting invasion and metastasis in most cancers, exhibited a downregulation in metastatic breast cancers, which is validated by migration and invasion assay ${ }^{24}$. miR-9 up-regulation in breast cancer cells, directly targets $C D H 1$, the E-cadherin-encoding mRNA, leading to increased cell motility and invasiveness ${ }^{25}$. Interestingly, miR-378(??) was recognized as a molecular switch in cancer cell bioenergetics pathway, also known as the Warburg effect, by the regulation of ERBB2 expression ${ }^{26}$. On the other hand, increasing the expression of few tumor suppressor miRNAs can alleviate development of breast tumors $^{27}$. For example, the expression of tumor suppressor miR-127 down-regulates the expression profile of protooncogene BCL6, a potential target of miR-12728.

\section{Colorectal cancer}

The major reason for the failure of treating advanced colorectal cancer is chemoresistance ${ }^{29}$. Several miRNAs have been found to be associated (miR-192, miR-215, miR-140, miR-129, let-7, miR-181b, miR-200 s) with chemoresistance by regulating key cell death pathways such as apoptosis and autophagy. Several important miRNA were described that regulate targets such as $\mathrm{Bcl} 2$, thymidylate synthase, dihydrofolate reductase, histone deacetylase, and E2F. For example, miR-215 was identified to suppress the expression of both thymidylate synthase and dihydrofolate reductase ${ }^{30}$. In addition, the expression of miR-215 was directly regulated by p53. The expression of miR-215 was significantly associated with colorectal cancer patient survival. Another miRNA, miR-140, was found to modulate chemosensitivity by suppressing HDAC 4 expression, and the levels of miR-140 and miR-215 were elevated in colon cancer stem cells ${ }^{31}$. Furthermore, miR-194 was identified to regulate BMI-1 protein expression (BMI-1 is involved in epithelial to- mesenchymal transition $)^{32}$. Moreover, miR-502 regulates autophagy in colon cancer by targeting Rab1B ${ }^{33}$. Taken together, these miRNAs can be utilized in predicting patient's prognosis and survival.

miRNA as biomarker for diabetes mellitus (DM)

The circulating miRNA (serum and plasma) characterizes a unique form of disease initiation and development. Due to a variety of pathogenesis of diverse types of $\mathrm{D}$, the differential regulatory roles of miRNA leading to disease outcomes, including ? ?-cell deficiency and insulin resistance, have recently been defined. In type $1 \mathrm{DM}$, the role of miRs in controlling ?-cell genesis, ?-cell death (miR-21), insulin production (miR-30d, miR204, miR-124a) and ?] ?-cell mass balance (miR-375) or its susceptibility to immunemediated ?-cell destruction has been described ${ }^{34-39}$. In regard to type $2 \mathrm{DM}$, insulin resistance miRNAs, the key regulators for homeostasis, has been characterized based on the differential expression of the candidate miRNA in insulin targeted DM patients. Some of the insulin sensitivity related miRNA in adipocytes (miR-21, miR-29, miR-93, miR-103, miR-143, miR-320), muscle (miR-1, miR-106b, miR-133a, miR-223), and liver (let-7, miR-130a-3p, miR-143, miR-181a, miR-802) were essential in maintaining physiological homeostasis and energy balance ${ }^{40-42}$. Studies by Zampetaki et al. ${ }^{43}$ showed decreased levels of 10 miRNAs in plasma of diabetic patients (miR15a, miR-20b, miR-21, miR-24, miR-126, miR-191, miR-197, miR-223, miR-320 and miR-486).

\section{miRNA as biomarker for neurodegenerative disease}

Neurodegenerative diseases include several central nervous system disorders characterized by the progressive loss of neural tissues and CNS damage. Hence, early diagnosis is essential to maximize the effectiveness of disease-modifying therapies. In recent years, much effort has been taken to recognize the neuropathological, biochemical, and genetic biomarkers of the diseases so that the diagnosis could be established in the earlier stages. The biomarkers for Alzheimer's, Parkinson disease and other neurodegenerative diseases must be reliable and specific, and they should be useful in guiding us to make more accurate diagnosis and better treatment of the diseases.

\section{Alzheimer's disease (AD)}

miRNAs has been demonstrated as potential non-invasive biomarkers from blood and serum for a wide variety of human pathologies ${ }^{44}$. A deregulation of miRNA expression might be involved in neurological dysfunction or neurodegenerative processes. Interestingly, Liang et al. ${ }^{45}$ showed a specific expression signature pattern of brain and blood mononuclear cells as a useful biomarker for $\mathrm{AD}$ and other neurological diseases. Though altered miRNA expression patterns have been extensively investigated in AD patients' tissue samples or cell cultures ${ }^{46}$, yet less information on circulating miRNAs in $A D$ is known. A recent serum profiling of $A D$ 
patients provided first evidence that expression changes of circulating miRNAs may be valuable biomarkers for $\mathrm{AD}^{47}$. Recently, Leidinger et al. ${ }^{48}$ has reported 140 unique differentially expressed miRNAs between $\mathrm{AD}$ patients and healthy controls. Further, Lugli et al. ${ }^{49}$ has reported that the expression of microRNAs in plasma fraction enriched in exosomes showed twenty miRNAs with significant differences in the AD group (miR-23b-3p, miR-24-3p, miR-29b-3p, miR-125b-5p, miR-138-5p, miR-139-5p, miR-141-3p, miR-150-5p, miR-152-3p, miR-185-5p, miR-338-3p, miR-342-3p, miR-342-5p, miR-548at-5p, miR-659-5p, miR-3065-5p, miR-3613-3p, miR-3916, miR-4772-3p, miR-5001-3p). Recently, in CIDRF, one of the Scientist, Dr.Doulathunnisa had been working on to determine the effect of artificial sweeteners on miRNA expression that leads to the regulation of $\mathrm{AD}$.

\section{Schizoprenia}

A hemizygous deletion of a 1.5-3-Mb region of chromosome 22 can lead to the 22q11 deletion syndrome (22q11DS), which is characterized by multiple physical and psychiatric abnormalities. A previous study determined that $~ 30 \%$ of 22q11DS patients may develop schizophrenia ${ }^{50}$. miR25 and miR-185 are regulators of the sarco/endoplasmic reticulum $\mathrm{Ca}^{2+}$ ATPase (SERCA2), which is responsible for loading $\mathrm{Ca}^{2+}$ into the endoplasmic reticulum. Earls et al., found that miR-25 and miR-185 were depleted in mouse models of 22q11DS and restoration of these miRNAs to presynaptic neurons rescued the long-term potentiation of DGCR $8^{+/-}$mice ${ }^{51}$. The authors concluded that miRNAdependent SERCA2 dysregulation is a pathogenic event in 22q11DS and schizophrenia. Gardiner et al. ${ }^{52}$ investigated the expression profile of miRNA in PBMCs of 112 patients and identified 83 miRNAs that were significantly downregulated in the schizoaffective group on chromosome 14q32. Similarly, Lai et al..$^{53}$ identified a signature of seven miRNAs in an initial cohort of 30 patients with schizophrenia which included the upregulated miR-34a, miR-449a, miR-564, miR-548d, miR-572 and miR-652, and downregulated miR-432.

\section{miRNA as biomarkers for pulmonary disease}

Tuberculosis (TB) is a chronic infectious disease caused by Mycobacterium tuberculosis. Some studies have identified a group of miRNAs that are expressed specific and play regulatory roles in the interaction between $M$.tuberculosis and host cells; these include miR-223, miR-144, and miR-421. In a collaborative project between CIDRF and Pulmonary medicine, sputum and serum miR-144 levels among newly diagnosed pulmonary tuberculosis patients before and after treatment will be correlated.

\section{Use of miRNA as Prognostic markers}

microRNAs (miRNAs), endogenous small noncoding RNAs, are found to be detected in plasma, serum, saliva and urine. The diagnosis and prognosis of different pathological conditions are linked with individual miRNAs and their signature patterns ${ }^{54}$. Tumor-specific miRNAs have been identified in cancer patients ${ }^{55}$. Plasma miRNAs derived from tissues have been used as biomarkers for injury ${ }^{56}$. Also, alterations in circulating miRNAs have been found in cardiovascular diseases, diabetes melletius, neurodegenerative disease, as well as autoimmune diseases ${ }^{57-59}$. A list of $\mathrm{miR}$ as prognostic markers has been listed in Table 1.

\section{Table 1: List of miRNA as prognostic marker in cancerous tissues.}

\begin{tabular}{|c|c|c|}
\hline miRNA & Cancer type & References \\
\hline $\begin{array}{l}\operatorname{miR}-205 \text {; Let-7F } \\
\operatorname{miR}-145 \\
\operatorname{miR}-124 \\
\operatorname{miR}-23 b\end{array}$ & Ovarian cancer & $\begin{array}{l}\text { Zheng et al., } 2013^{67} \\
\text { Zhang et al., } 2013^{68} \\
\text { Wang et al., } 2013^{69}\end{array}$ \\
\hline $\begin{array}{l}\mathrm{miR}-200 \text { and } \mathrm{miR}-203 \text {, miR-30a and miR- } \\
155\end{array}$ & Metastatic breast cancer & Madavan et al., $2016^{70}$ \\
\hline $\begin{array}{l}\text { let-7c, let-7e, miR-30c, miR-622, and miR- } \\
1285 \text {, miR-141 and miR-375; exosomal miR- } \\
1290 \text { and miR-375 }\end{array}$ & $\begin{array}{l}\text { castration-resistant prostate } \\
\text { cancer }\end{array}$ & Huang et al., $2015^{71}$ \\
\hline
\end{tabular}




\begin{tabular}{|c|c|c|}
\hline $\begin{array}{l}\text { miR-148b, miR-376c, miR-409-3p, and } \\
\text { miR-801 }\end{array}$ & Primary breast cancer & Cuk et al., $2013^{72}$ \\
\hline $\begin{array}{l}\mathrm{miR}-25 \text { and } \mathrm{miR}-223, \mathrm{miR}-574-5 \mathrm{p} \text { and } \mathrm{miR}- \\
\text { 1254, miR-21, miR-126, miR-486-5p, and } \\
\text { miR-210 }\end{array}$ & non-small-cell lung cancer & Shen et al., $2011^{73}$ \\
\hline $\begin{array}{l}\text { miR-17-3p and miR-92, miR-601 and miR- } \\
\text { 760, miR-221, miR-29b }\end{array}$ & Colorectal cancer & $\begin{array}{l}\text { Wang et al., } 2012^{74} \text {, Inoue } \\
\text { et al., } 2015^{75}\end{array}$ \\
\hline $\operatorname{miR}-328$ & Glioblastoma & Yuan et al., $2016^{76}$ \\
\hline
\end{tabular}

\section{Cardiovascular disease}

Circulating miRNAs have been investigated as possible novel prognostic markers for prediction of CHD progression and adverse outcomes. Several studies have explored associations between cardiac function and miRNAs as a predictor of future adverse outcomes in CHD. miR-134, -198 , and -370 , a miR signature pattern was detected by Hoekstra et al. ${ }^{60}$ in view to differentiate unstable from stable angina pectoris suggesting a potential prognostic tool in cardiovascular disease. miR-370 has been identified as a possible prognostic biomarker in an earlier study in mice. Also, increased miR-370 expression levels had been found in response to induced ischemia ${ }^{61}$. Zampetaki et al. ${ }^{62}$ has reported three signature miRNAs ((miR-126, miR-197 and miR-223) for the prediction of myocardial infarction (MI), where miR-126 levels are found to be positive, while the other miRs are inversely associated with future MI.

\section{Diabetes mellitus}

miRNAs are being available as biomarkers for monitoring of disease onset and progression. They have a great tendency to serve as accurate diagnostic and prognostic markers, as well as being viable therapeutic targets for treating diabetes complications. A large number of specific miRNAs have appeared as major regulators of particular aspects of disease pathologies including diabetes complications.

\section{Cancer}

As an important factor in tumorigenesis, microRNAs (miRNAs) are anticipated as potential biomarkers for early cancer detection and accurate prognosis as well as targets for more efficient treatment. Alterations in miRNAs lead to resistance for anticancer drugs and are well-known to be dysregulated in cancer; current literature revealed that miRNA levels in biological samples may be interrelated with chemotherapy response. miRNA expression profiles vary between normal tissues and cancerous cells derived from the same organ, and also between cancer types miRNAs can either function as oncogenes nor tumor suppressors, thereby leading to various pathways in tumorigenesis ${ }^{63}$. They may be used for prognostic purposes and they also constitute novel targets for cancer treatment. Recently, the evidence for the roles of miRNAs in determining drug sensitivity/ resistance has been emerging. miRNA that can be used as prognosis in different types of cancer have been listed in table.

Also, human mesenchymal stromal/stem cells generally have the potency to differentiate into various mesenchymal cell lineages that makes them a challenging cell source for the use in tissue repair strategies. Georgi et al ${ }^{64}$ has recently investigated that profiling of hMSC donors for specific panel of miRNAs could serve as a prognostic marker for selecting donors with high differentiation potential to improve hMSC-based tissue repair approach. Peripheral blood miRNA has shown expression patterns that serve as a challenging prognostic tool in primary CNS lymphoma patients ${ }^{65}$.

\section{miRNA profiling}

miRNA expression profiling is mainly been done to identify miRNAs that play a critical role in organismal development, establishment and maintenance of tissue differentiation as biomarkers and serve as reagents for the reprogramming of cell fate in stem cell applications. miRNA profiling has attracted many researchers to work in various research areas of biology and medicine. miRNA profiling is defined as the measurement of the relative abundance of a cohort of miRNAs, ranging from a group of several miRNAs of specific biological interest to comprehensive profiling of all miRNAs in a given species (typically numbering in the several 


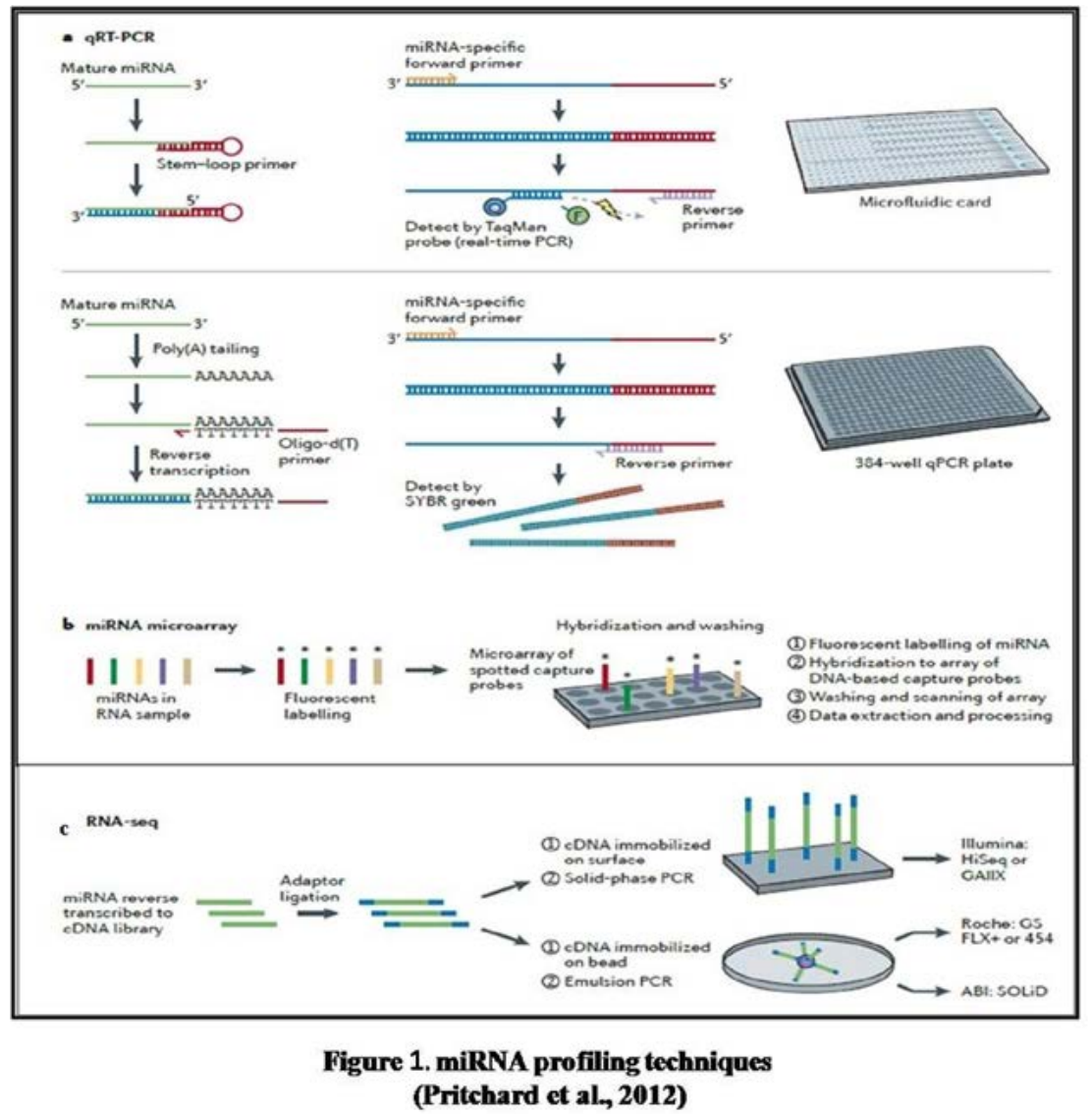

hundred $)^{66}$. Assaying such small RNA molecules poses some inherent challenges, but technological advances in recent years have overcome many of these barriers, and a wide range of approaches and platforms is now available for miRNA profiling (Figure 1).

\section{Quantitative reverse transcription PCR-based methods.}

One major approach relies on reverse transcription of miRNA to cDNA, followed by qPCR with realtime monitoring of reaction product accumulation (known as 'real- time PCR'). An appealing aspect of this approach is the ease of incorporation into the workflow for laboratories that are familiar with realtime PCR. In order to scale this approach for miRNA profiling, reactions are carried out in a highly parallel, high-throughput form (that is, hundreds of qRT-PCR reactions measuring different miRNAs using the same reaction conditions). Two common strategies used for priming the reverse transcription reaction to generate cDNA are enzymatic addition of a poly (A) tail and generation of a reverse transcription primer binding site using a stem-loop primer. A hurdle in performing highly parallel qRT-PCR is that optimal reaction conditions may vary substantially between
miRNAs owing to sequence-specific differences in primer annealing. Although different vendors have sought to solve this problem using various approaches, one effective strategy has been the incorporation of locked nucleic acids (LNAs) into primers to standardize optimal miRNA primer hybridization conditions for the hundreds of PCR assays that are to be run simultaneously.

\section{Hybridization-based methods}

The first method to perform parallel analysis of large number of miRNAs is done by microarrays, where different approaches for fluorescent labelling of the miRNA in a biological sample for subsequent hybridization to DNA- based probes on the array will be performed. One commonly used labelling approach is the enzymatically catalysed ligation of a fluorophoreconjugated nucleotide or short oligonucleotide to the terminal 3? $\mathrm{?}-\mathrm{OH}$ of the miRNA using T4 RNA ligase. Another enzymatic-labelling approach involves 3 ? tailing of the miRNA (for example, with poly (A), following which a fluorophore-conjugated oligonucleotide may be ligated using a splinted ligation. Alternative chemical approaches to miRNA labelling exist, that includes chemical alkylation-based labelling 
along the miRNA and approaches based on platinum coordination chemistry with nucleic acids. It should also be kept in mind that other cellular RNAs, in addition to miRNAs, may be labelled by both enzymatic and chemical approaches, which can contribute to background signal as well as to cross-hybridization with specific miRNA probes.

\section{RNA-sequencing}

The advent of next-generation sequencing platforms has enabled a third major approach for miRNA expression profiling. The general approach begins with the preparation of a small RNA cDNA library from the RNA sample of interest, followed by the 'massively parallel' sequencing of mil- lions of individual cDNA molecules from the library. Bioinformatic analysis of the sequence reads identifies both known and novel miRNAs in the data sets and provides relative quantification using a digital approach (that is, the number of sequence reads for a given miRNA relative to the total reads in the sample is an estimate of relative abundance of the miRNA). The major advantages of next-generation sequencing for miRNA profiling are detection of both novel and known miRNAs and precise identification of miRNA sequences (for example, RNA- seq can readily distinguish between miRNAs that differ by a single nucleotide, as well as isomiRs of varying length). However, it should be noted that RNA-seqbased miRNA-profiling studies typically identify a plethora of small RNAs of novel sequence (that is, putative miRNAs), but not all of these may be bona fide miRNAs. Potential limitations of next-generation sequencing include the high cost, although this is dropping with the introduction of newer versions of the instruments, and the use of DNA 'barcoding', which permits multiplexing of many samples in a single run.

\section{Conclusion}

Taking into account of the use of miRNA as biomarkers for clinical diagnostics and prognostics values outlined in this review will allow for more precise understanding for their potential use in clinical applications. Further elucidation of miRNA biogenesis and functionality will enable the development of more specific and sensitive assays. Enhancing the art of performing research and implying its application in clinical set-up will lead to exciting novel gene regulators. Also, their specific functions will augment the opportunities to safely pursue them as therapeutic modalities.

\section{REFERENCES}

1. miRBase Sequence Database. Available online: http://microrna.sanger.ac.uk/sequences (accessed on 23 May 2014).

2. Kozomara A, Griffiths-Jones S. miRBase: Integrating microRNA annotation and deep-sequencing data. Nucleic Acids Res. 2011; 39:D152-57.

3. Ambros V, Bartel B, Bartel DP, Burge CB, Carrington JC et al. A uniform system for microRNA annotation. RNA. 2003; 9:277-9.

4. Wightman B, Ha I, Ruvkun G. Posttranscriptional regulation of the heterochronic gene lin-14 by lin-4 mediates temporal pattern formation in C. elegans. Cell. $1993 ; 75: 855-62$.

5. Lee RC, Feinbaum RL, and Ambros V. The C. elegans heterochronic gene lin-4 encodes small RNAs with antisense complementarity to lin-14. Cell. 1993; 75:843-54.

6. Griffiths-Jones S, Saini HK, van Dongen S, Enright AH. miRBase: tools for microRNA genomics. Nucleic Acids Res. 2008; 36:D154-8.

7. Kim VN, Han J, Siomi MC. Biogenesis of small RNAs in animals. Nat Rev Mol Cell Biol. 2009; 10:126-39.

8. Baskerville S, Bartel DP. Microarray profiling of microRNAs reveals frequent coexpression with neighboring miRNAs and host genes. RNA. 2005; $11: 241-7$.

9. Landgraf P, Rusu M, Sheridan R, Sewer A, lovino N, et al. A mammalian microRNA expression atlas based on small RNA library sequencing. Cell. 2007 ; $129: 1401-14$.

10. Saini HK, Griffiths-Jones S, Enright AJ. Genomic analysis of human microRNA transcripts. Proc Natl Acad Sci USA. 2007; 104:17719-24.

11. Borchert GM, Lanier W, Davidson BL. RNA polymerase III transcribes human microRNAs. Nat Struct Mol Biol. 2006; 13:1097-101.

12. Bartel DP. MicroRNAs: target recognition and regulatory functions. Cell. 2009; 136:215-33.

13. Khvorova A, Reynolds A, Jayasena SD. Functional siRNAs and miRNAs exhibit strand bias. Cell. 2003; 115:209-16.

14. Cheloufi S, Dos Santos C0, Chong MM, Hannon GJ. A dicer-independent miRNA biogenesis pathway that requires Ago catalysis. Nature. $2010 ; 465: 584-9$.

15. Zhao Y, Samal E, Srivastava D. Serum response factor regulates a muscle-specific microRNA that targets Hand2 during cardiogenesis. Nature. 2005 ; $436: 214-20$.

16. Li X. MiR-375, a microRNA related to diabetes. Gene. 2014; 533:1-4.

17. Chen CZ, Li L, Lodish HF, Bartel DP. MicroRNAs modulate hematopoietic lineage differentiation. Science. 2004; 303:83-6.

18. Giraldez AJ, Cinalli RM, Glasner ME, Enright AJ, Thomson JM, et al. MicroRNAs regulate brain morphogenesis in zebrafish. Science. 2005; 308:833-8.

19. Döhner H, Stilgenbauer S, Benner A, Leupolt E, Kröber A, et al. Genomic aberrations and survival in chronic lymphocytic leukemia. N Engl J Med. 2000; 343:1910-6.

20. Mishra PJ. MicroRNAs as promising biomarkers in cancer diagnostics. Biomark Res. 2014; 2:19.

21. Mishra PJ, Merlino G. MicroRNA reexpression as differentiation therapy in cancer. J Clin Invest. 2009; 119:2119-23.

22. Stupp R, Hegi ME, Mason WP, van den Bent MJ, Taphoorn MJ et al. Effects of radiotherapy with concomitant and adjuvant temozolomide versus radiotherapy alone on survival in glioblastoma in a randomised phase III study: 5-year analysis of the EORTC-NCIC trial. Lancet 0ncol. 2009; 10:459-66.

23. Hua D, Mo F, Ding D, Li L, Han X et al. A catalogue of glioblastoma and brain MicroRNAs identified by deep sequencing. 0MICS. 2012; 16:690-9.

24. Ma L, Teruya-Feldstein J, Weinberg RA. Tumour invasion and metastasis initiated by microRNA-10b in breast cancer. Nature. 2007; 449:682-8

25. Ma L, Young J, Prabhala H, Pan E, Mestdagh P, et al. miR-9, a MYC/MYCN-activated microRNA, regulates E-cadherin and cancer metastasis. Nat Cell Biol. 2010 ; 12:247-56.

26. Eichner LJ, Perry MC, Dufour CR, Bertos N, Park M, et al. miR-378(??) mediates metabolic shift in breast cancer cells via the PGC-1ß/ERRy transcriptional pathway. Cell Metab. 2010; 12:352-61.

27. Lowery AJ, Miller N, McNeill RE, Kerin MJ. MicroRNAs as prognostic indicators and therapeutic targets: potential effect on breast cancer management. Clin Cancer Res. 2008; $14: 360-5$. 
28. Saito Y, Liang G, Egger G, Friedman JM, Chuang JC, et al. Specific activation of microRNA-127 with downregulation of the proto-oncogene BCL6 by chromatin-modifying drugs in human cancer cells. Cancer Cell. 2006; 9:435-43.

29. Ju J, Jiang J, Fesler A. miRNA: the new frontier in cancer medicine. Future Med Chem. 2013; 5:983-5.

30. Song B, Wang Y, Titmus MA, Botchkina G, Formentini A et al. Molecular mechanism of chemoresistance by miR-215 in osteosarcoma and colon cancer cells. Mol Cancer. 2010; 9:96.

31. Karaayvaz M, Pal T, Song B, Zhang C, Georgakopoulos P, et al. Prognostic significance of miR-215 in colon cancer. Clin Colorectal Cancer. 2011; 10:340-7.

32. Zhai H, Karaayvaz M, Dong P, Sakuragi N, Ju J. Prognostic significance of miR-194 in endometrial cancer. Biomark Res. 2013; 1:12.

33. Zhai H, Song B, Xu X, Zhu W, Ju J. Inhibition of autophagy and tumor growth in colon cancer by miR-502. Oncogene. 2013; 32: 1570-9.

34. Lynn FC, Skewes-Cox P, Kosaka Y, McManus MT, Harfe BD, et al. MicroRNA expression is required for pancreatic islet cell genesis in the mouse. Diabetes. 2007; 56: 2938-45.

35. Poy MN, Hausser J, Trajkovski M, Braun M, Collins S, et al. miR-375 maintains normal pancreatic alpha- and beta-cell mass Proc Natl Acad Sci USA. 2009; 106: 5813-8.

36. Tang X, Muniappan L, Tang G, Ozcan S. Identification of glucose-regulated miRNAs from pancreatic beta cells reveals a role for miR-30d in insulin transcription. RNA. 2009; 15: 287-93.

37. Mi QS, He HZ, Dong Z, Isales C, Zhou L. MicroRNA deficiency in pancreatic islet cells exacerbates streptozotocin-induced murine autoimmune diabetes Cell Cycle. 2010 9: 3127-9.

38. Ruan Q, Wang T, Kameswaran V, Wei Q, Johnson DS, et al. The microRNA-21-PDCD4 axis prevents type 1 diabetes by blocking pancreatic beta cell death Proc Natl Acad Sci USA. 2011; 108: 12030-5.

39. Xu G, Chen J, Jing G, Shalev A. Thioredoxin-interacting protein regulates insulin transcription through microRNA-204. Nat Med. 2013; 19: 1141-6.

40. Ling HY, Hu B, Hu XB, Zhong J, Feng SD, et al. MiRNA-21 reverses high glucose and high insulin induced insulin resistance in 3T3-L1 adipocytes through targeting phosphatase and tensin homologue. Exp Clin Endocrinol Diabetes. 2012; 120:553-9.

41. Chen YH, Heneidi S, Lee JM, Layman LC, Stepp DW, et al. miRNA-93 inhibits GLUT4 and is overexpressed in adipose tissue of polycystic ovary syndrome patients and women with insulin resistance. Diabetes. 2013; 62: 2278-86.

42. Frost RJ, Olson EN. Control of glucose homeostasis and insulin sensitivity by the Let-7 family of microRNAs. Proc Natl Acad Sci USA. 2011; 108:21075-80.

43. Zampetaki A, Kiechl S, Drozdov I, Willeit P, Mayr U, et al. Plasma microRNA profiling reveals loss of endothelial miR-126 and other microRNAs in type 2 diabetes. Circ Res. 2010; 107: 810-17.

44. Wang K, Yuan Y, Cho JH, McClarty S, Baxter D, et al. Comparing the MicroRNA Spectrum between Serum and Plasma. PLoS One. 2012; 7:e41561.

45. Liang Y, Ridzon D, Wong L, Chen C. Characterization of microRNA expression profiles in normal human tissues. BMC Genomics. 2007; 8:166.

46. Long JM, Lahiri DK. MicroRNA-101 downregulates Alzheimer's amyloid-beta precursor protein levels in human cell cultures and is differentially expressed. Biochem Biophys Res Commun. 2010; 404: 889-95.

47. Geekiyanage H, Jicha GA, Nelson PT, Chan C. Blood serum miRNA: non-invasive biomarkers for Alzheimer's disease. Exp Neurol. 2011; 235: 491-6.

48. Leidinger P, Backes C, Deutscher S, Schmitt K, Mueller SC, et al. A blood based 12-miRNA signature of Alzheimer disease patients. Genome Biol. 2013 ; 14:R78.

49. Lugli G, Cohen AM, Bennett DA, Shah RC, Fields CJ et al. Plasma Exosomal miRNAs in Persons with and without Alzheimer Disease: Altered Expression and Prospects for Biomarkers. PLoS One. 2015; 10:e0139233.

50. Williams NM. Molecular mechanisms in 22q11 deletion syndrome. Schizophr Bull. 2011; 37:882-9.

51. Earls LR, Fricke RG, Yu J, Berry RB, Baldwin LT, et al. Age-dependent microRNA control of synaptic plasticity in $22 q 11$ deletion syndrome and schizophrenia. J Neurosci. 2012; 32: 14132-44.

52. Gardiner E, Beveridge NJ, Wu JQ, Carr V, Scott RJ, et al. Imprinted DLK1-DI03 region of 14q32 defines a schizophrenia-associated miRNA signature in peripheral blood mononuclear cells. Mol Psychiatry. 2012; 17:827-40.

53. Lai CY, Yu SL, Hsieh MH, Chen CH, Chen HY, et al. MicroRNA expression aberration as potential peripheral blood biomarkers for schizophrenia. PLoS 0ne. 2011; 6:e21635.

54. Pritchard CC, Cheng HH, Tewari M. MicroRNA profiling: approaches and considerations. Nat Rev Genet. 2012; 13:358-69.

55. Zubakov D, Boersma AW, Choi Y, van Kuijk PF, Wiemer EA, et al. MicroRNA markers for forensic body fluid identification obtained from microarray screening and quantitative RT-PCR confirmation. Int J Leg Med. 2010; 124:217-26.

56. Jeyaseelan K, Lim KY. MicroRNA expression in the blood and brain of rats subjected to transient focal ischemia by middle cerebral artery occlusion. Stroke. 2008; 39:959-66.

57. Tanaka M, Oikawa K. Down-regulation of miR-92 in human plasma is a novel marker for acute leukemia patients. PLoS 0ne. 2009; 4:e5532.

58. Wang K, Zhang S. Circulating microRNAs, potential biomarkers for drug-induced liver injury. Proc Natl Acad Sci USA. 2009; 106: 4402-07.

59. Wang GK, Zhu JQ. Circulating microRNA: A novel potential biomarker for early diagnosis of acute myocardial infarction in humans. Eur Heart J. 2010; 31:659-66.

60. Tijsen AJ, Creemers EE. MiR423-5p as a circulating biomarker for heart failure. Circ Res. 2010; 106: 1035-9.

61. Igaz I, Szőnyi M, Varga P, Topa L. Potential relevance of microRNAs in the diagnostics of inflammatory bowel diseases. Orv Hetil. 2014; 155 : 487-91.

62. Hoekstra M, van der Lans CA, Halvorsen B, Gullestad L, Kuiper J, et al. The peripheral blood mononuclear cell microRNA signature of coronary artery disease. Biochem Biophys Res Commun 2010; 394:792-7.

63. $\mathrm{Xu} \mathrm{CF}, \mathrm{Yu} \mathrm{CH}$, Li YM. Regulation of hepatic microRNA expression in response to ischemic preconditioning following ischemia/reperfusion injury in mice. 0mics 2009; 13:513-20.

64. Zampetaki A, Willeit P, Tilling L, Drozdov I, Prokopi M, et al. Prospective study on circulating MicroRNAs and risk of myocardial infarction. J Am Coll Cardiol 2012; 60:290-9

65. Croce CM, Calin GA. miRNAs, cancer, and stem cell division. Cell. 2005, 122:6-7.

66. Georgi N, Taipaleenmaki H, Raiss CC, Groen N, Portalska KJ, et al. MicroRNA Levels as Prognostic Markers for the Differentiation Potential of Human Mesenchymal Stromal Cell Donors. Stem Cells Dev. 2015; 24:1946-55.

67. Zheng $\mathrm{H}$, Zhang L, Zhao Y, Yang D, Song F, et al. Plasma miRNAs as diagnostic and prognostic biomarkers for ovarian cancer. PLoS One. 2013 ; 8:e77853.

68. Zhang H, Wang Q, Zhao Q, Di W. MiR-124 inhibits the migration and invasion of ovarian cancer cells by targeting SphK1J. Ovarian Res. $2013 ; 6: 84$.

69. Wang YQ, Guo RD, Guo RM, Sheng W, Yin LR. MicroRNA-182 promotes cell growth, invasion, and chemoresistance by targeting programmed cell death 4 (PDCD4) in human ovarian carcinomas J Cell Biochem. 2013; 114: 1464-73.

70. Madhavan D, Peng C, Wallwiener M, Zucknick M, Nees J, et al. Circulating miRNAs with prognostic value in metastatic breast cancer and for early detection of metastasis. Carcinogenesis. 2016 Jan 19.

71. Huang X, Yuan T, Liang M, Du M, Xia S, et al. Exosomal miR-1290 and miR-375 as prognostic markers in castration-resistant prostate cancer. Eur Urol. 2015; 67:33-41.

72. Cuk K, Zucknick M, Heil J, Madhavan D, Schott S, et al. Circulating microRNAs in plasma as early detection markers for breast cancer. Int J Cancer. 2013; $132: 1602-12$.

73. Shen J, Todd NW, Zhang H, Yu L, Lingxiao X, et al. Plasma microRNAs as potential biomarkers for non-small-cell lung cancer. Lab Invest. 2011; 91:579-87.

74. Wang Q, Huang Z, Ni S, Xiao X, Xu Q, et al. Plasma miR-601 and miR-760 are novel biomarkers for the early detection of colorectal cancer. PLoS One. $2012 ; 7: e 44398$.

75. Inoue A, Yamamoto H, Uemura M, Nishimura J, Hata T, et al. MicroRNA-29b is a Novel Prognostic Marker in Colorectal Cancer. Ann Surg Oncol. 2015;22 Suppl 3:S1410-8

76. Yuan J, Zheng Z, Zheng Y, Lu X, Xu L, et al. microRNA-328 is a favorable prognostic marker in human glioma via suppressing invasive and proliferative phenotypes of malignant cells. Int J Neurosci. 2016; 126:145-53. 